\title{
The Wheat Microbiome Under Four Management Strategies, and Potential for Endophytes in Disease Protection
}

\author{
Kristi Gdanetz, Department of Plant Biology, Michigan State University; and Frances Trail, ${ }^{\dagger}$ Department of Plant \\ Biology and Department of Plant, Soil, and Microbial Sciences, Michigan State University, East Lansing, MI 48824
}

Accepted for publication 29 September 2017.

\begin{tabular}{|c|c|}
\hline \multicolumn{2}{|c|}{ ABSTRACT } \\
\hline $\begin{array}{l}\text { Manipulating plant-associated microbes to reduce disease } \\
\text { or improve crop yields requires a thorough understanding of } \\
\text { interactions within the phytobiome. Plants were sampled from } \\
\text { a wheat/maize/soybean crop rotation site that implements four } \\
\text { different crop management strategies. We analyzed the fungal and } \\
\text { bacterial communities of leaves, stems, and roots of wheat } \\
\text { throughout the growing season using } 16 \mathrm{~S} \text { and fungal internal } \\
\text { transcribed spacer } 2 \text { rRNA gene amplicon sequencing. The most }\end{array}$ & $\begin{array}{l}\text { prevalent operational taxonomic units (OTUs) were shared across } \\
\text { all samples, although levels of the low-abundance OTUs varied. } \\
\text { Endophytes were isolated from plants, and tested for antagonistic } \\
\text { activity toward the wheat pathogen Fusarium graminearum. } \\
\text { Antagonistic strains were assessed for plant protective activity in } \\
\text { seedling assays. Our results suggest that microbial communities } \\
\text { were strongly affected by plant organ and plant age, and may be } \\
\text { influenced by management strategy. }\end{array}$ \\
\hline
\end{tabular}

The basis for interactions among microorganisms as communities are beginning to be elucidated, including bacteria, fungi, protists, and viruses growing on and within host organisms. Microbes interact with plants in a variety of ways: pathogens utilize the plants as a food source, symbiotic mycorrhizae and rhizobia exchange nutrients with their hosts, endophytes live inside plant cells asymptomatically and some species can provide protection to the plants against harsh environmental conditions (Rodriguez et al. 2009). Yet, basic knowledge about the structure of microbial communities across plant organs is still lacking.

Wheat is a staple food globally, and is one of the most commonly grown crops, with approximately two billion bushels produced in the United States annually (USDA 2016). The fungal pathogen Fusarium graminearum has resulted in devastating yield losses, estimated at \$2.491 to 7.67 billion between 1993 and 2001 (McMullen et al. 2012). There are few control options as fungicides have low efficiency against $F$. graminearum and there are no strongly resistant varieties (reviewed by Wegulo et al. 2015). One potential method that can contribute to an integrated approach to control is manipulation of plant microbial communities to suppress pathogen populations. The most practical method to achieve this goal involves colonization with parasitic or competitive endophytes that will kill or displace pathogens of interest.

${ }^{\dagger}$ Corresponding author: F. Trail; E-mail: trail@msu.edu

*The $\boldsymbol{e}$-Xtra logo stands for "electronic extra" and indicates that 13 supplementary figures and 8 supplementary tables are published online.

(C) 2017 The American Phytopathological Society
The term endophyte is used to describe microbial organisms that spend the majority or entirety of their life cycle living within a host plant (Rodriguez et al. 2009). Endophytic fungi have been documented to benefit their plant hosts in diverse conditions. They can improve salt and heat tolerance in wild grasses (Rodriguez et al. 2008). In wheat, improved germination rates have been attributed to endophytes (Hubbard et al. 2012), and protective effects of endophytes against Stagonospora infection have been documented (Sieber et al. 1988). Recently, bacterial endophytes have been shown to reduce disease and mycotoxin production by pathogens in millet (Mousa et al. 2016). Identification of wheat endophytes may provide novel strains to improve crop health and reduce disease.

Previous studies of wheat microbiomes have largely focused on identifying microbes in the roots or rhizosphere (for example, Hartmann et al. 2014; Mahoney et al. 2017; Ofek et al. 2013; Yin et al. 2017), while noticeably fewer studies have focused on aboveground organs (Granzow et al. 2017; Huang et al. 2016; Karlsson et al. 2017). To our knowledge, there are no published studies which have surveyed the entire wheat microbiome, including both above- and below-ground plant organs, with high throughput sequencing techniques. Here we classify the bacterial and fungal microbiomes of three wheat organs (stems, leaves, and roots) grown under four land management strategies (conventional tillage, no-till, low input, and organic). Microbial communities were dependent on type of plant organ, and community composition changed as plants matured. We then used the wheat microbiome analysis as the context for identifying and testing potential biocontrol strains isolated from the experimental plots for protective abilities against $F$. graminearum seedling damping-off. 


\section{MATERIALS AND METHODS}

Microbiome sample collection. Wheat plants were collected from the Michigan State University (MSU) W.K. Kellogg Biological Station (KBS) Long-Term Ecological Research (LTER) main crop rotation site located in Hickory Corners, Michigan (42.411085'N 85.377078'W; https://lter.kbs.msu.edu/). The soils of the KBS-LTER site are Typic Hapludalfs of the Kalamazoo (fineloamy, mixed, mesic) and Oshtemo (coarse-loamy, mixed, mesic) series, developed on glacial outwash (Crum and Collins 1995). Soil series were mapped onto the sample site using the USDA Official Soil Series Descriptions: https://soilseries.sc.egov.usda.gov/osdname. aspx. All wheat/maize/soybean rotation plots were of the soil series Kalamazoo, except plots T1-R3, T3-R3, and T4-R4 which were Oshtemo. The site has been under continuous wheat/maize/soybean rotations since 1993 (Robertson 2015) and is organized in randomized, replicated 1-ha plots under four land management strategies with six replicates of each conventional till, no-till, reduced chemical inputs with an alfalfa cover crop, and organic with an alfalfa cover crop (Supplementary Fig. S1). In the fall of 2012, seeds of soft red winter wheat, variety 25R39 (Pioneer Hi-Bred International, Inc., Johnston, IA), treated with Gaucho fungicide (Bayer Corp., Pittsburgh, PA), were planted in plots of all management strategies, except the organic plots, which were sown with untreated seeds of the same variety. Weeds were controlled by tilling in organic plots and chemically controlled in the other plots.

Plants were collected at the following Zadoks stages (Zadoks et al. 1974) and dates: stage 30 (vegetative) on 1 May 2013; stage 45 (late boot, early flowering) on 30 May 2013; and stage 83 (early seed development) on 5 July 2013. Six random plants, with intact roots, were removed from each of the 24 plots for microbiome analysis. Plants were bagged in pairs; henceforth each pair of plants was treated as one biological replicate. Roots and aboveground tissues were placed in separate sterile sample collections bags (Nasco Whirl-Pak, Fort Atkinson, WI) and maintained on ice during transport. Plants were stored at $-80^{\circ} \mathrm{C}$ then lyophilized. Lyophilized tissue was stored at room temperature under a desiccant until processed for DNA isolation.

rRNA gene amplification and sequencing. Approximately $50 \mathrm{mg}$ of $0.2 \mathrm{~mm}^{2}$ pieces of leaf, stem, or root tissues (fine and thick) were transferred into ClavePak 1.1-ml tubes (Denville Scientific, Holliston, MA) containing 5/32" (3.97 mm) stainless steel ball bearings (Grainger, Lansing, MI). DNA extractions were performed in triplicate for each biological replicate using the Mag-Bind Plant DNA Plus Kit (Omega Bio-tek, Norcross, GA) following the manufacturer's protocols with a Retsch Oscillating Mill M400 (Verder Scientific, Newtown, PA) and a KingFisher Flex (ThermoFisher Scientific, Waltham, MA). Phusion High Fidelity DNA Polymerase (New England Biolabs, Ipswich, MA) was used to amplify the $16 \mathrm{~S}$ V4 and internal transcribed spacer 2 (ITS2) rRNA gene regions of bacteria and fungi, respectively. PCR amplification of each sample was performed in triplicate, PCR products were pooled and purified with Wizard Gel and PCR Clean-up Kit (Promega, Madison, WI). Amplicons were sequenced at the MSU Research Technology Support Facility (East Lansing, MI) using a dualindex barcode strategy (Kozich et al. 2013) and Illumina MiSeq $2 \times$ $250 \mathrm{bp}$ chemistry. Barcodes were used to distinguish between samples from replicate plots of each management strategy, growth stage, and plant organ. Reads are available in NCBI Small Read Archive under BioProject PRJNA356450 and accession number SRP102192.

Bioinformatics. The USEARCH pipeline (version v8.1.1861) was used for quality filtering, trimming, operational taxonomic unit (OTU)clustering, and chimera detection. The cluster threshold was set to $97 \%$ similarity (Edgar 2010; Edgar and Flyvbjerg 2015; Edgar et al. 2011). The Ribosomal Database Project Naive Bayesian Classifier was used for taxonomic assignment with the $16 \mathrm{~S}$ and UNITE fungal training sets (Cole et al. 2013; Kõljalg et al. 2013; Wang et al. 2007). OTUs belonging to Archaea, Plantae, and Protista were discarded. Samples were normalized with variance-stabilizing normalization and significant OTUs were identified using the 'DESeq2' package (Love et al. 2014; McMurdie and Holmes 2014). To determine within-sample diversity, alpha diversity statistics were calculated with the 'phyloseq' package (McMurdie and Holmes 2013; Rideout et al. 2014), significance was tested by analysis of variance (ANOVA) and Tukey's honest significant difference in the $\mathrm{R}$ statistical computing environment. To determine between-sample diversity, ordination analyses on Bray-Curtis and Jaccard distances, permutational multivariate ANOVA (PERMANOVA) to test community centroids and homogeneity of variance to test community variance, were calculated using the 'adonis' and 'betadisp' functions in the 'vegan' package (Oksanen et al. 2016). Graphs were generated with 'ggplot2' (Wickham 2009). Analyses were completed using R version 3.3.2 (R Core Team 2016).

Microbial isolation and identification. Two additional plants for microbe isolations were collected at each growth stage, following the methods as described above. Plants were stored at $4{ }^{\circ} \mathrm{C}$, and processed as described below within $48 \mathrm{~h}$ of sample collection. Endophytic fungi were isolated as previously described (Arnold et al. 2000) with slight modifications: ten $2 \mathrm{~mm}^{2}$ pieces of tissue were removed from roots, stems, or leaves and surface sterilized by soaking in $10 \%$ sodium hypochlorite with $0.1 \%$ Tween 80 for 2 min, followed by a rinse in $70 \%$ ethanol for $2 \mathrm{~min}$, and a quick rinse in sterile distilled water. Surface sterilized tissue was incubated at room temperature in Nutrient Broth Yeast Extract agar medium (Suay et al. 2000) or $2 \%$ malt extract agar (MEA; Amresco, Solon, OH) appended with ampicillin at $50 \mu \mathrm{g} / \mathrm{ml}$ to reduce contaminating bacteria. Emerging fungi were transferred singly to MEA and subcultured twice to obtain a homogeneous culture.

Endophytic bacteria were isolated as follows: roots or intact aboveground tissues of vegetative stage plants were surface sterilized as described above. For plants from the two later growth stages, leaves, roots, and stems were individually surface sterilized and ground in $0.85 \%$ aqueous $\mathrm{NaCl}$ with glass beads in a mortar and pestle (Compant et al. 2011). Three $10 \times$ serial dilutions of the extraction wash were generated and duplicates of each dilution were streaked onto R2A medium (Reasoner and Geldreich 1985) supplemented with cycloheximide at $40 \mu \mathrm{g} / \mathrm{ml}$ to reduce contamination by eukaryotic microbes.

Nonendophytic microbes were isolated by cutting tissue into small fragments, approximately $5 \mathrm{~mm}^{2}$, and transferring them to selective media. MEA or Rose Bengal agar medium supplemented with ampicillin at $50 \mu \mathrm{g} / \mathrm{ml}$ was used to capture a diverse population of fungi. Fungal colonies were subcultured as described above. To select for bacteria, R2A medium supplemented with cycloheximide at $40 \mu \mathrm{g} / \mathrm{ml}$. Bacterial colonies were restreaked at least three times, and single colonies were isolated to ensure cultures were genetically homogeneous.

Fungal isolates used in plant protection assays and morphotypes of isolates used in in vitro competition assays were identified by sequencing of the full ITS rRNA gene region. DNA extraction was performed on lyophilized mycelium of isolates used in plant protection assays and isolates of representative morphotypes with the Extract-N-Amp kit (Sigma-Aldrich, St. Louis, MO) as modified by Bonito et al. (2011). The ITS region was amplified with the ITS1F and ITS4 primer pair (Supplementary Table S1) and Phusion High Fidelity DNA Polymerase, following manufacturer's recommendations (New England Biolabs, Ipswich, MA). PCR products were purified with EXOSAP-IT (Affymetrix, Santa Clara, CA) and sequenced at the MSU Research and Technology Support Facility. Sequences were identified via BLAST matches to the NCBI database (Altschul et al. 1990).

In vitro competition assays. A Michigan isolate of $F$. graminearum (PH-1, NRRL \#31084, FGSC \#9075, Trail and Common 2000) was used in all in vitro competition and plant protection assays. Petri dishes 
(35-mm-diameter) containing MEA were simultaneously inoculated with $F$. graminearum and the antagonistic fungus at opposite poles. Bacterial isolates were streaked across the midline of one-half of the dishes $24 \mathrm{~h}$ after inoculation of $F$. graminearum. Fungal interactions were recorded starting at $48 \mathrm{~h}$ after inoculation. Interactions were classified based on observed phenotypes for up to 10 days postinoculation (Supplementary Fig. S2).

Plant protection assays. Endophytic isolates that exhibited inhibitory activity in vitro were used in planta to determine if endophytes could protect against seedling blight. Wheat seeds (cultivar Wheaton) were surface sterilized in $95 \%$ ethanol for $10 \mathrm{~s}$, rinsed in sterile distilled water for $10 \mathrm{~s}$ followed by a 3-min wash in $5 \%$ sodium hypochlorite, and three rinses in sterilized distilled water. Seeds were germinated on 6-cm MEA plates colonized with 2- to 3-day-old cultures of isolates of the endophytic fungi, as described in Hubbard et al. (2012). After 3 days, endophyte-inoculated seeds were transferred to $50 \mathrm{ml}$ cone-tainers (Steuwe and Sons, Inc., Tangent, OR) with potting mix (Suremix Perlite, Michigan Grower Products, Inc., Galesburg, MI). Plants were challenged by inoculation with $F$. graminearum with the addition of colonized agar; $1 / 6$ of a $10-\mathrm{cm}$ diameter Petri dish containing synthetic nutrient-poor agar (SNA) medium (Baldwin et al. 2010). Control plants were potted with sterile SNA (Supplementary Fig. S3). The assay was replicated three times, independently, each isolate was tested with 10 plants per replicate. Isolates were scored for plant protective abilities, based on disease incidence, as calculated by lesion presence at the base of the stem or deceased plants.

\section{RESULTS}

Microbiome composition across plant organs and land management strategies. The ITS2 rRNA gene sequencing of all samples, from wheat organs at three growth stages and under four management strategies, generated 31,507,778 reads. Of 216 samples, sequences from 214 resulted in usable sequences that passed quality filtering. Sequence processing was performed with the USEARCH pipeline and identified 3,164 ITS2 OTUs at 97\% similarity for clustering (Supplementary Table S2).

Taxonomic composition changed across growth stage, and root communities contained more unique members than phyllosphere communities. Recently, several usages for "phyllosphere" have appeared in the literature. For clarity, in this publication we will use "phyllosphere" to refer to aerial parts of the plant, as previously defined (Ruinen 1956; Vorholt 2012). During the vegetative growth stage under all management strategies, approximately $40 \%$ of fungal OTUs were classified as Dothideomycetes, followed by a decrease in abundance at flowering, and then an increase to 50 to $90 \%$ of all observed sequences at seed development (Fig. 1, Supplementary Table S3). Dothideomycetes were classified as members of the Pleosporaceae, Phaeosphaeriaceae, or Leptosphaeriaceae, but many
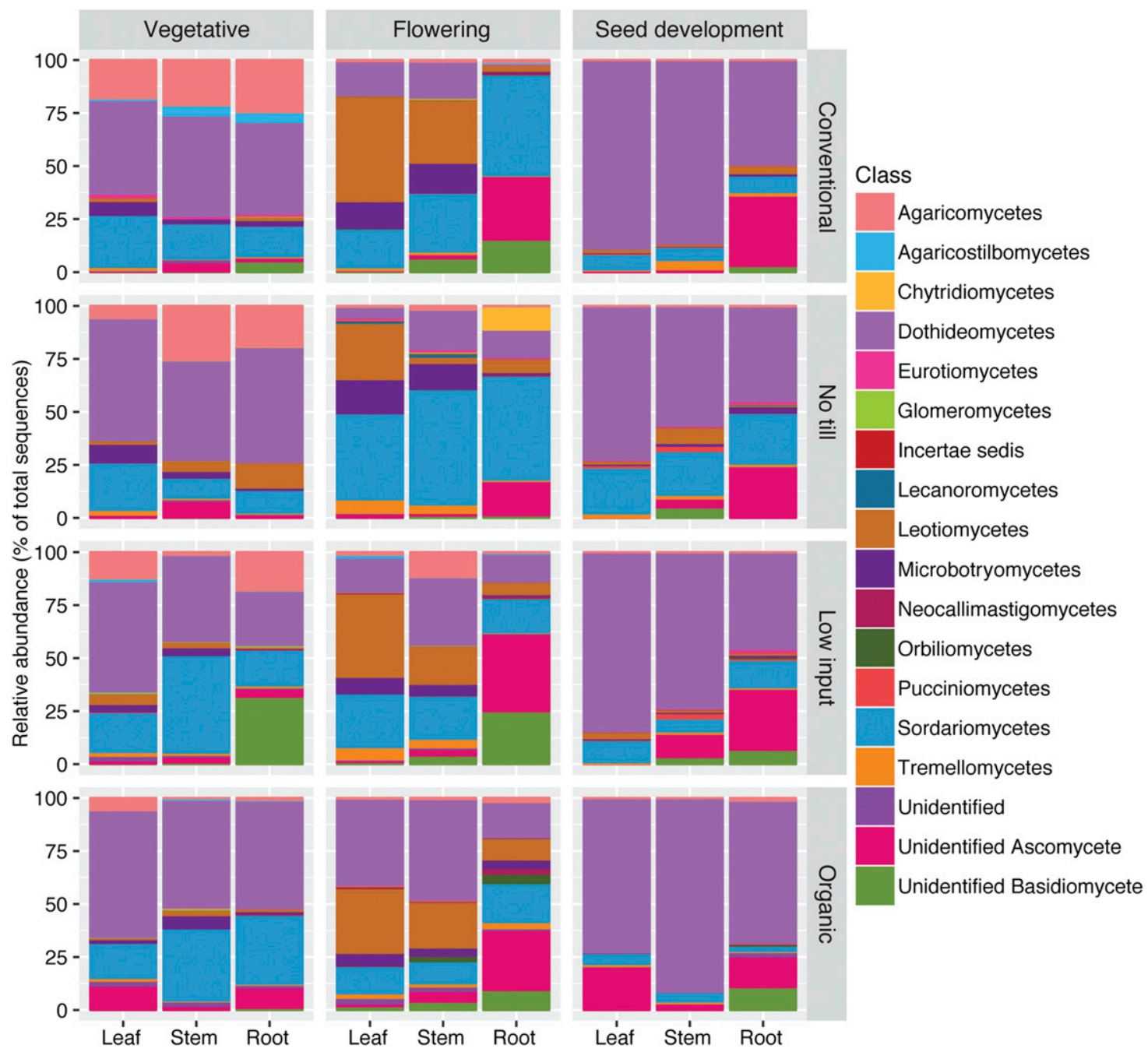

Fig. 1. Class-level relative abundance of fungal communities across growth stage, plant organ, and crop management strategies. 
Dothideomycete OTUs remained unclassified at the family level (Supplementary Fig. S4). Across the growing season, we observed a decrease from vegetative to flowering stages in the relative abundance of low-abundance OTUs, such as Tremellomycetes, Microbotryomycetes, Leotiomycetes, and Agaricomycetes. Agaricomycetes populations were largely members of the families Ceratobasidiaceae and Marasmiaceae. Microbotryomycetes populations were dominated by the family Leucosporidiaeae (Supplementary Fig. S5). At flowering, we observed a higher proportion of Eurotiomycetes in leaf samples, specifically the families Herpotrichiellaceae and Massariaceae, compared with vegetative and seed development stages. Also at flowering, leaf and stem samples were dominated by the Leotiomycetes, specifically the Erysiphaceae (Supplementary Fig. S6).

The majority of OTUs, across all samples, belonged to a few groups (Dothideomycetes, Sordariomycetes, and Agaricomycetes); however, abundances of some OTUs differed across management strategy. Notably, in conventional and no-till plots, we observed a greater abundance of Microbotryomycetes in leaves and stems (Fig. 1). Roots, compared with leaves or stems at all growth stages and under all management strategies, had higher numbers of unidentified OTUs, with nearly $50 \%$ of the relative abundance of OTUs from conventional and low input plots (Fig. 1). The no-till samples had more unique OTUs, and approximately $25 \%$ of the observed OTUs belonged to the Sordariomycetes. Sordariomycete populations were largely composed of members of the Nectriaceae and
Lasiosphaeriaceae across all management strategies, except the Glomerellaceae dominated the leaves and stems of low input and organic management types during seed development. Pairwise comparison of unique OTUs in the roots revealed that Periconia sp. was enriched in no-till and organic plots when compared with all other management strategies. Tetracladium sp. was enriched in all low-input plots, and Fusarium sp. was enriched in all organic plots (Supplementary Fig. S7). The same analysis of the phyllosphere samples showed enrichment of Fusarium sp. in organic and no-till plots, enrichment of Ceratobasidium sp. in low-input plots when compared with all other management strategies. Puccinia sp. was enriched in conventional, low input, and organic plots when these management strategies were compared with no-till (Supplementary Fig. S8).

The 16S rRNA gene sequencing generated 28,082,995 sequences that clustered into 7,906 OTUs. All 216 samples generated high quality sequences. Proteobacteria was the most abundant bacterial Phylum, followed by Bacteroidetes and Firmicutes (Supplementary Table S4). At the Class level, Alphaproteobacteria, Actinobacteria, Gammaproteobacteria, Deltaproteobacteria, Planctomycetia, and Sphingobacteria were the most abundant OTUs (Fig. 2; Supplementary Table S5).

Fungal alpha diversity was generally consistent across organs and management strategies, then slightly decreased during seed development (Fig. 3A). The fungal diversity of plant organs, as calculated



Fig. 2. Class-level relative abundance of bacterial communities across growth stage, plant organ, and crop management strategies. 
by the Shannon Index $\left(H^{\prime}\right)$, was not significantly different during vegetative or flowering growth stages. But at the seed development stage, $H^{\prime}$ 'of stems was significantly lower than roots from the organic plots $(P \leq 0.05$; Fig. 3A; Supplementary Table S6). This correlates with the large increase in the dominance of Dothideomycetes in all plant organs as the most abundant OTUs during seed development. In contrast to the fungal samples, the bacterial alpha diversity in roots was significantly higher than in stems and leaves when compared $(P \leq 0.05)$ across growth stages and management strategies, but stems and leaves were not significantly different from each other (Fig. 3B).

To determine if beta diversity of microbial communities differed, ordination analysis was conducted within management types and organs. As stated by Kelly et al. (2015), 90\% power for PERMANOVA tests can be achieved with five independent samples in microbiome
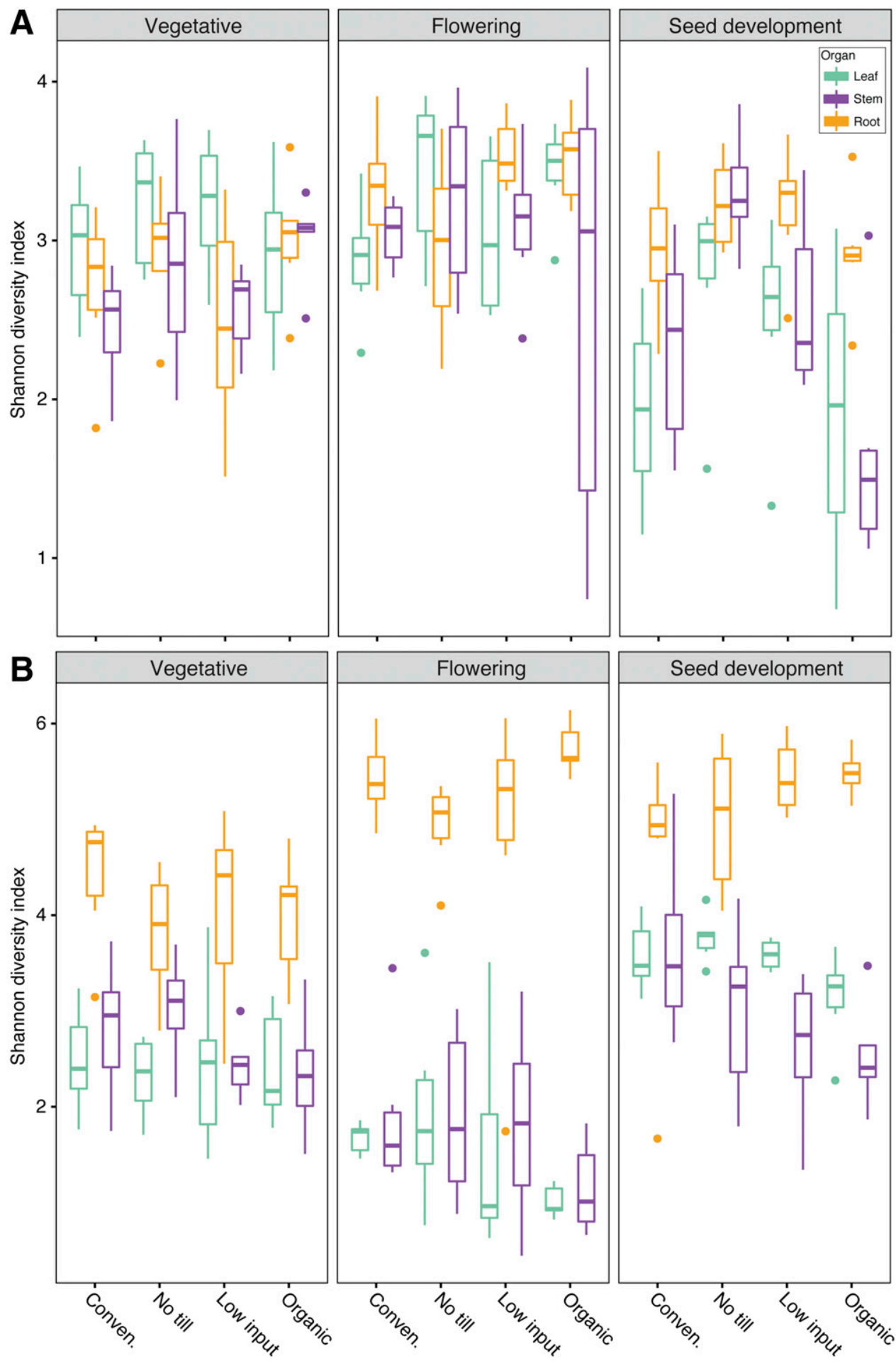

Fig. 3. Alpha diversity of A, fungi and B, bacteria found across all samples estimated by Shannon diversity index. Data are represented by six replicates from each stage-management-organ combination. Center line of boxes represents median of samples. The upper and lower sides of the boxes represent the third and first quartiles, respectively. Whiskers represent \pm 1.5 times the interquartile range. Data points beyond whiskers represent outliers. Analysis of variance and Tukey's honest significant difference were used to test significance $(P<0.05)$. Conven $=$ conventional. 
studies; here we tested groups of six independent samples. PERMANOVA tests showed that within each management strategy, fungal communities from different growth stages $(P<0.001)$ and organs $(P<0.05)$ had significantly different centroids (Fig. 4A; Supplementary Table
S7). Tests of homoscedasticity for growth stage were not significant, but were significant for organ in no-till and low input plots $(P<0.05)$. Fungal communities differ at each growth stage, and communities may differ across wheat organ. Centroids of bacterial
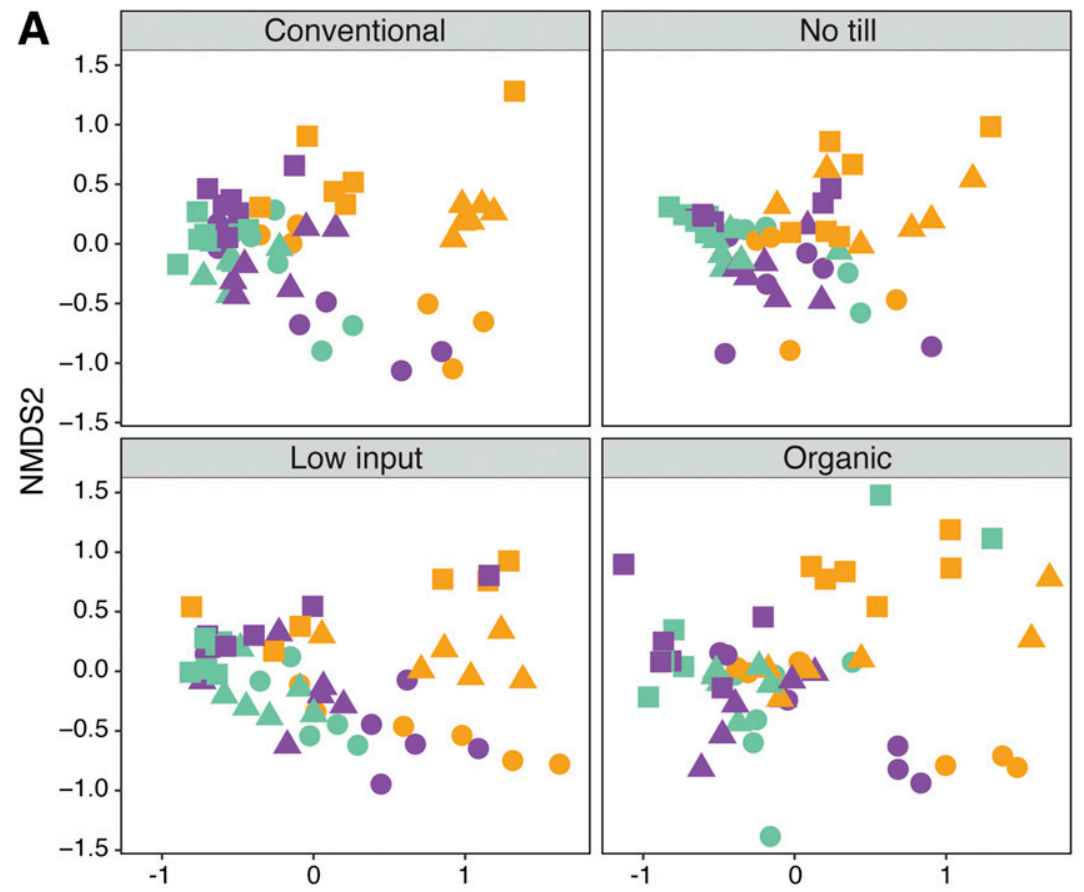

B
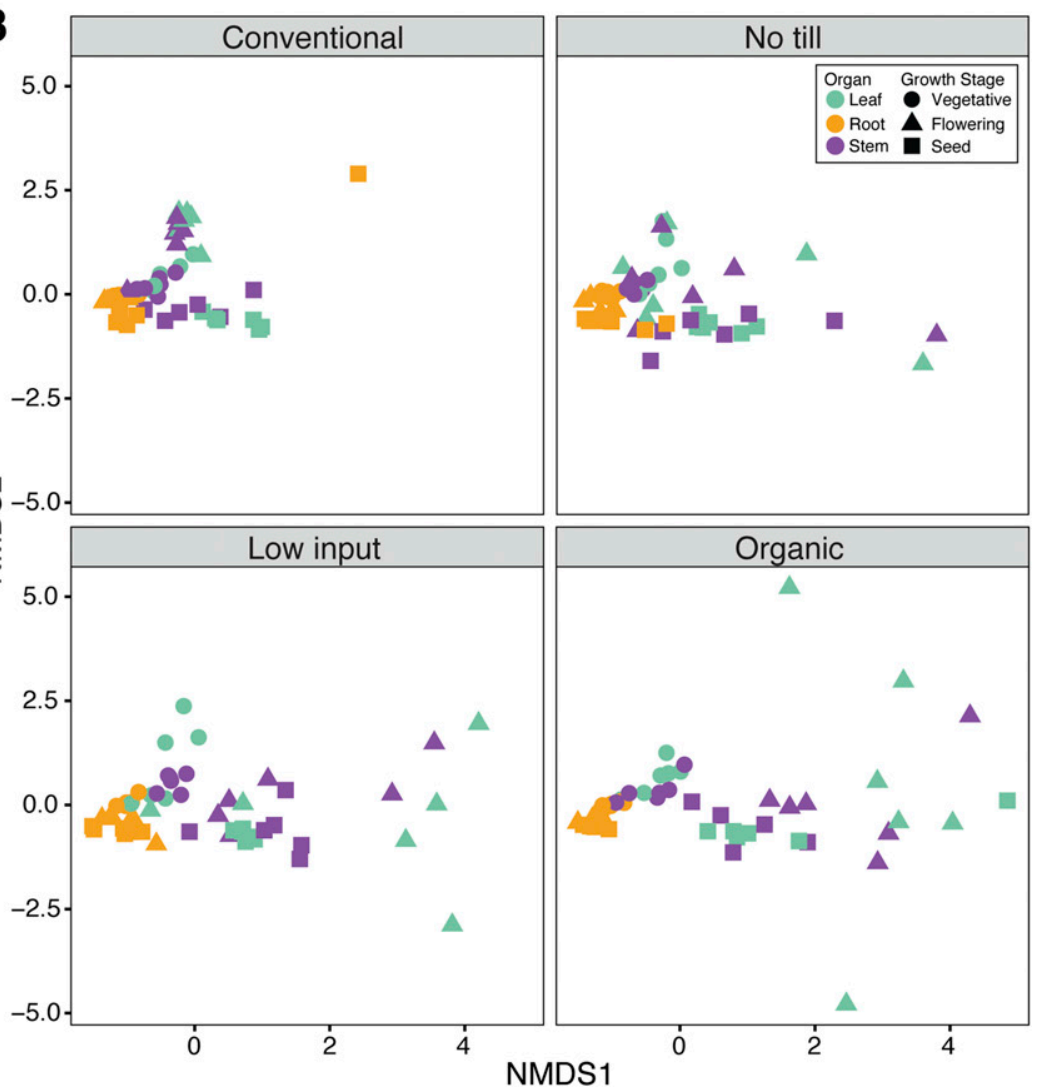

Fig. 4. Effect of management strategies on beta diversity of $\mathbf{A}$, fungal and $\mathbf{B}$, bacterial communities originating from plant organs. Nonmetric multidimensional scaling (NMDS) calculated by Bray-Curtis distance. Difference between centroids were tested using permutational multivariate analysis of variance and homoscedasticity to test variance (Oksanen et al. 2016). Stress values of fungal data (A), which reveal goodness of fit, were $0.115,0.117$, 0.117 , and 0.121 for conventional, no till, low input, and organic NMDS plots, respectively. Stress values of bacterial data (B) were $0.086,0.092,0.086$, and 0.113 for conventional, no till, low input, and organic NMDS plots, respectively. 
communities were significantly different for organ and growth stage $(P \leq 0.001)$ (Fig. 3B). Dispersions tests were significant for organs within all management strategies except no-till $(P \leq 0.01)$, and were significant for growth stages within all management strategies except conventional $(P \leq 0.05)$. The differences in group dispersions may cause observed clustering of bacterial communities by organ.

In contrast to the analysis of communities under different management strategies, PERMANOVA tests within each organ, showed fungal communities from different growth stages and management strategies had significantly different centroids $(P \leq 0.01)$ (Supplementary Fig. S9). Homoscedasticity tests on management strategy were not significant in any organ, but were significant for growth stage in stems $(P \leq 0.001)$. The fungal communities of all organs except stems, differ across management strategies and at each growth stage. Bacterial communities had significantly different centroids for management strategy and growth stage $(P \leq 0.05)$. Homoscedasticity tests were significant for growth stage $(P \leq 0.01)$ and management strategy in leaves $(P \leq 0.05)$. The observed differences in bacterial communities across growth stage may be due to differences in group dispersions, but it appears that management strategy affects the bacterial communities within different plant organs.

Microbe isolations and In vitro assays. A total of 1,634 fungal and 1,112 bacterial isolates were cultured from all wheat organs and management strategies across the three growth stages (Table 1). A subset of these isolates, 711 fungi and 715 bacteria were screened with an in vitro confrontation assay paired with $F$. graminearum. Based on observed interaction phenotypes, five categories were identified and all isolates used for the in vitro confrontation assay were placed in one of the five categories (Table 2). Type 1: Zone of inhibition between colonies, excessive pigment production by $F$. graminearum. Type 2: Zone of inhibition between colonies, no $F$. graminearum pigment observed in inhibition zone. Type 3: No zone of inhibition, mycelium deadlock between fungal colonies, or hyphal proliferation at fungal-bacterial colony interface. Type 4: $F$. graminearum hyphae grew over top of competing microbe. Type 5: Hyphae of competing fungus grew over top $F$. graminearum

TABLE 1

Microbe isolates recovered from wheat plants

\begin{tabular}{lcc}
\hline \multirow{2}{*}{ Growth stage } & \multicolumn{2}{c}{ Number isolated $^{\mathrm{a}}$} \\
\cline { 2 - 3 } Early vegetative & Fungi & Bacteria \\
\hline Boot & 456 & 510 \\
\hline Kernel development & 527 & 211 \\
\hline $\begin{array}{l}\text { a Numbers presented here are totals and do not account for duplicates } \\
\text { of morphotypes. }\end{array}$ & 651 & 391 \\
\end{tabular}

colony, or competing bacterial colony covered more than $50 \%$ of the petri dish. The fungal isolates within each of these categories were then grouped into morphotypes, isolates of similar morphological appearance (Table 2). Morphotypes were identified as Alternaria sp., Bipolaris sp., Cochliobolus sp., Fusarium sp., Colletotrichum sp., Talaromyces sp., Trichoderma sp., Parastagnospora sp., Penicillium sp., among others (Supplementary Table S8).

Greenhouse assay and isolate identification. Endophytic fungi (78 strains) that generated Type 1 interactions during in vitro confrontation assays were tested in a seedling assay. Fifteen of these strains showed biocontrol activity during an initial test, and these strains were pursued further. Many of the strains were identified as species known to be saprotrophs, weak grass pathogens, or pathogens of other plant species (strains and ID numbers can be found in Table 3). Positive controls (plants inoculated with $F$. graminearum in the absence of an endophyte) had an average $82 \%$ disease in the seedling assay (Fig. 5). Negative controls (plants not inoculated with an endophyte and without $F$. graminearum in the soil) had an average of $3 \%$ disease. The positive and negative controls were significantly different from each other $(\alpha=0.05)$ (Fig. 5). Endophyte inoculated seedlings challenged with $F$. graminearum in soil had a 10 to $41 \%$ reduction in disease compared with the positive control (Fig. 5). Strain 40 had significantly reduced disease compared with the positive control $(\alpha=0.05)$. All other endophyte inoculated plants, besides strain 40, had reduced disease, but due to high variance of the means the disease reduction was not significant. Two endophyte strains, 11 and 34, had higher or nearly equal disease rates in control plants when compared with $F$. graminearum-challenged plants (Fig. 5), indicating that these endophyte strains may be pathogens of wheat.

Fungal microbiome data were mined for OTUs closely related to the genera of the isolates used in the greenhouse assay. Alternaria sp. was found in nearly all samples, with the highest abundances in leaf samples (Supplementary Fig. S10). Microdochium sp. and Fusarium sp. were the next most abundant OTUs, with highest abundances specifically in root samples at the flowering and seed development growth stages (Supplementary Fig. S11). Phoma sp. were most abundant during seed development in conventional and no-till plots, and Talaromyces sp. were most abundant during flowering, but both Phoma sp. and Talaromyces sp. were found in relatively low abundances across multiple samples (Supplementary Fig. S12). Aspergillus sp. and Penicillium sp. were found in very few samples, namely the roots of no-till plants during flowering and vegetative stages, respectively (Supplementary Fig. S13).

\section{DISCUSSION}

One of the proposed applications of microbiome research is the manipulation of microbial communities to reduce pathogen pressure and increase yield. Before this can successfully occur, the composition

TABLE 2

Results of in vitro coculture competition assay with Fusarium graminearum

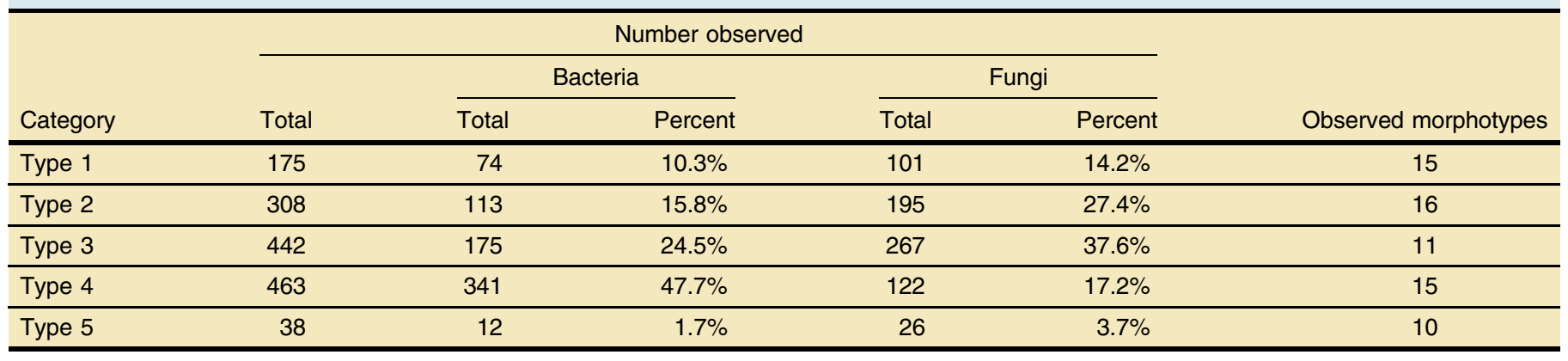


of a plant-associated community that can induce these effects must be determined. Toward this goal, the present study provides the first indepth analysis of the wheat microbiome-phyllosphere, roots, and rhizosphere.

Contrary to our expectations, management strategy did not have a strong influence on plant microbial communities. Previous studies

TABLE 3

Identification of most protective fungal strains used in greenhouse plant assays

\begin{tabular}{ll}
\hline Strain ID & ID by full-length internal transcribed spacer locus \\
\hline 11 & Microdochium bolleyi \\
\hline 30 & Alternaria tenuissima \\
\hline 34 & Alternaria sp. \\
\hline 35 & Talaromyces trachyspermus \\
\hline 36 & Aspergillus niger \\
\hline 37 & Alternaria tenuissima \\
\hline 38 & Fusarium solani \\
\hline 40 & Fusarium sp. \\
\hline 44 & Fusarium sp. \\
\hline 45 & Penicillium reticulisporum \\
\hline 51 & Phoma sp. \\
\hline 57 & Phoma sp. \\
\hline 59 & Fusarium sp. \\
\hline 80 & Fusarium oxysporum \\
\hline
\end{tabular}

compared soil and root microbial communities across management strategies, such as conventional versus organic including wheat in the rotation. These studies have reached varied conclusions regarding the impact of management strategies on soil or plant associated microbial communities (Hartmann et al. 2014; Lenc et al. 2014; Li et al. 2012). Studies which found that management strategy influenced the microbial community compared sites in which the rotated crops differed across management strategy and/or compared sites which were separated by significant geographic distances (Hartmann et al. 2014; Lenc et al. 2014; Li et al. 2012; Rascovan et al. 2016). Similar to the present study, a previous study on the wheat phyllosphere used sites with a maximum distance of $10 \mathrm{~km}$ between fields of differing management strategies (Karlsson et al. 2017). The majority of the of the high-abundance OTUs from leaf microbiomes were detected across all fields and management strategies, but the authors found significant differences in lowabundance OTUs. Previous surveys of plant microbiomes comparing the same genotypes across multiple locations found that geography has a stronger influence on microbial community than management strategy or plant genotype (Chen et al. 2016; Copeland et al. 2015; Finkel et al. 2011; Peiffer et al. 2013). As discussed by Peay et al. (2016), decay-by-distance patterns for fungal communities are commonly observed, indicating geography and associated environmental factors impose strong effects on fungal community composition. These same factors would likely also impose these effects on the bacterial community.

The structure of the KBS-LTER site is nearly ideal for testing management effects. All of the plots are located within $2 \mathrm{~km}^{2}$ and have been planted with the same three-crop rotation for almost three decades. Studies of soil microbial communities conducted at the KBS-LTER site found similar results to the present study. For example, Lauber et al. (2013) sampled the $16 \mathrm{~S}$ soil community of

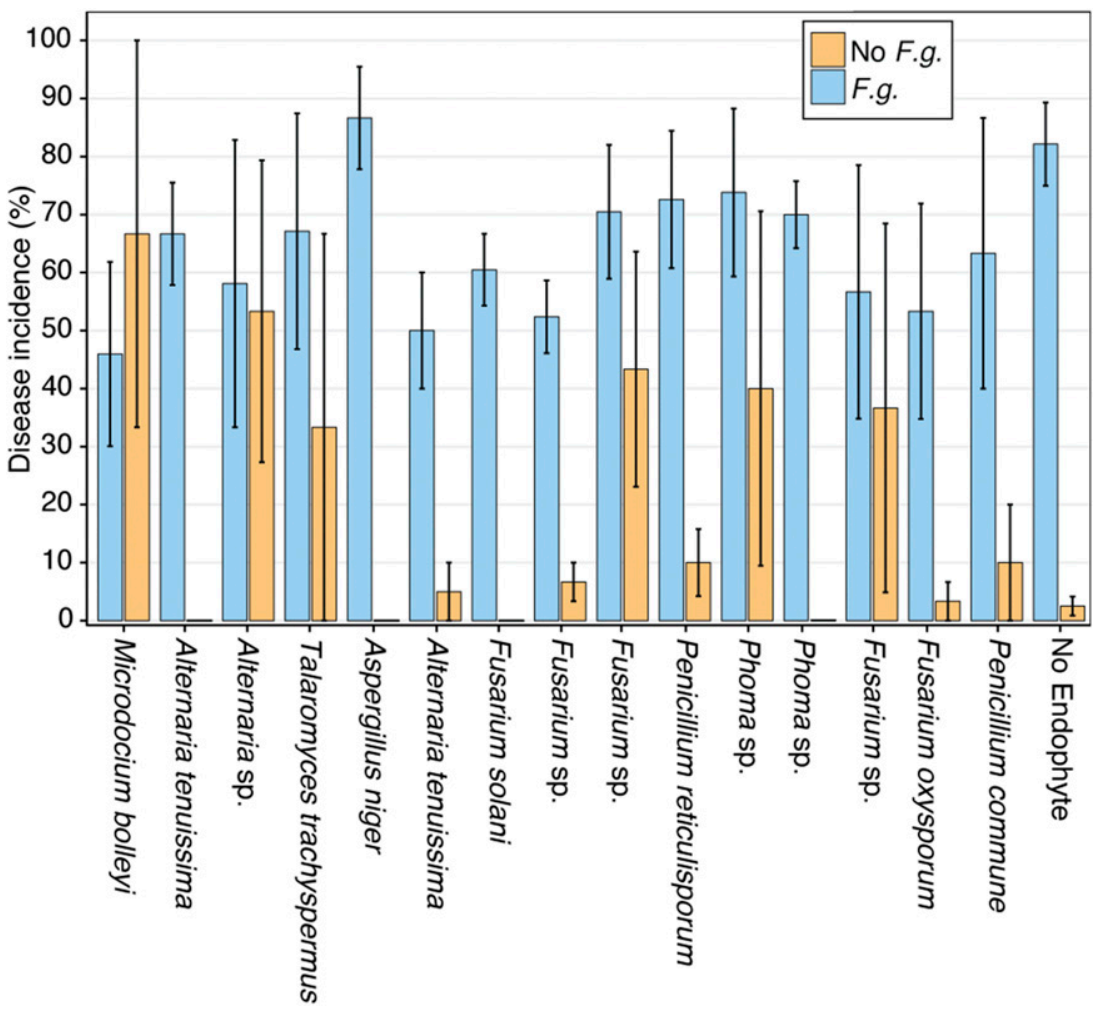

Fig. 5. Mean disease incidence (percentage of replicate) of endophyte-inoculated seedlings \pm standard error of the mean. Ten plants per replicate, three independent replicates. Analysis of variance and Tukey's honest significant difference were used to test significance $(P<0.05)$. F.g. $=F u s a r i u m$ graminearum. 
the conventional and low-input plots at the KBS-LTER. They observed similar community composition among management strategies, and also observed increased soil diversity throughout the growing season (Lauber et al. 2013). Xue et al. (2013) investigated the functional differences in soil communities of the KBS-LTER plots using the Geochip, an array based on genes involved in biogeochemical processes of soil microbes. They surveyed conventional, no-till, and low input plots, but found no significant differences in community function (Xue et al. 2013). Seed treatments could influence these outcomes. In our study, plots from three of the management strategies were planted with fungicide-treated seed, and the seed for the organic plots was not treated. This did not appear to affect the outcome, in that there were no major differences between the microbiomes of the plants in these plots. However, previous studies have shown that fungicide treatments affect rhizosphere and phyllosphere microbiomes of maize and soybean tested during the vegetative growth phase (Nettles et al. 2016). Further research is warranted to detect the impact of seed fungicides on the extant microbiome, and to determine the degree the fungicide, inherited microbes, or environment impact the microbiome of a mature plant. In the present study, the absence of strong management effects in any of the three growth stages, together with the survey of published data discussed above, suggest a strong influence of geographical factors on wheat microbiomes. However, it is likely that a combination of climate, cultivar, land use history, and management strategies all impact plant microbial communities in ways we may not be able to detect at this time.

Our study found that bacterial alpha diversity in roots was higher than in stems and leaves, whereas fungi show consistent diversity across plant organs. These results are consistent with previous studies showing soil and rhizosphere bacterial diversity were greater than diversity of the phyllosphere (Coleman-Derr et al. 2015; de Souza et al. 2016; Knief et al. 2011), and others showing rhizosphere and phyllosphere fungal diversity were similar (Coleman-Derr et al. 2015). The observed consistencies in fungal diversity across plant organs may be due to systemic colonization of crops by endophytic fungi, or due to aerial dispersal and subsequent phyllosphere colonization by fungal spores. Microclimate effects on plant organs that inhibit colonization by microbes may be one explanation for the low diversity of phyllosphere bacterial communities on wheat.

Our experimental design did not distinguish between epiphytic and endophytic microbes. Other studies that examined microbial communities of epiphytic and endophytic plant compartments found that the plant organs had a stronger effect than compartment on fungal community assembly (Coleman-Derr et al. 2015; de Souza et al. 2016). In contrast, the rhizosphere and the leaf episphere, had higher bacterial diversity than corresponding endophytic compartments (Coleman-Derr et al. 2015; de Souza et al. 2016). Thus, it is not surprising that microbial communities of stems and leaves in the present study were very similar. It is possible that in the rhizosphere and in the root endophytic compartment, the plant genotype imparts a greater selective force on community assembly (Coleman-Derr et al. 2015; Mendes et al. 2014; Ofek et al. 2013; Peiffer et al. 2013).

We observed increased microbial community diversity over the growing season. This is a consistent pattern found across other plant microbiome studies of cereals and fruits (Bakker et al. 2017; Donn et al. 2014; Shade et al. 2013). However, the observed increase in diversity is in contrast with the results of Copeland et al. (2015) who found a decrease in phyllosphere bacterial diversity throughout the growing season in beans and canola. The increase in diversity could be explained by ecological succession within the plant microbiome through the growing season, as emerging surfaces on crops bringing new habitats and an expansion of niche breadth. As plants grow and age, the community complexity and diversity would then increase. In addition, the observed increase in diversity could be a direct response to signals between the plants and microbes; that is, colonization by saprotrophic organisms that are able to colonize the plant host at earlier growth stages. The increased diversity could also reflect microbial responses to complex metabolites produced by mature plants. These interactions would not be limited to pathogens or saprotrophs. A more comprehensive study of the colonization of older plants is needed.

The bacterial communities of wheat at the KBS-LTER site were composed of common wheat- and plant-associated taxa. Previous wheat-microbiome studies, which were limited to studies of soils of wheat fields, roots, or rhizosphere, found similar fungal and bacterial community composition as the study presented here. Proteobacteria, Firmicutes, and Actinobacteria were the dominant bacterial taxa in the KBS-LTER plots, similar to other wheat rhizosphere studies (Donn et al. 2014; Hartmann et al. 2014; Lenc et al. 2014; Ofek et al. 2013). Dothideomycetes, Leotiomycetes, and Sordariomycetes were the most common fungal taxa observed in the present study as well as previous studies (Karlsson et al. 2017; Kwaśna et al. 2010; Lenc et al. 2014). Future studies are needed to investigate genotype-, inflorescence-, and developing seed-specific microbial communities.

The limited approaches available for control of $F$. graminearum suggest implementation of an integrated approach combining crop rotation and use of partially resistant varieties as the optimum control strategy. Biologicals used as soil or seed amendments would provide another tool to control a difficult disease. As reviewed recently, the phyllosphere microbiome contains organisms that influence plant defense and carbon cycling, among other functions (Bulgarelli et al. 2013; Rastogi et al. 2013; Vorholt 2012). We used damping-off of seedlings to test our strains for protective effects. In wheat seedlings, damping-off is currently controlled with fungicide-coated seeds (Wegulo et al. 2015), but fungicide resistance in $F$. graminearum is an increasing problem. We tested single protective strains, however multistrain protection from disease is likely to make a more robust application in the field, as has been reported (Slininger et al. 2010). We speculate that these tested strains, isolated in Michigan, are likely to be more successful protectors than products derived from strains native to other geographic regions, based on previous work with Trichoderma sp. biological controls (Chaverri et al. 2015; Grondona et al. 1997; Sharma et al. 2009) and aflatoxin control strains (Adhikari et al. 2016).

For more than 150 years, plant pathologists have shown us that individual microbes have adapted to specific niches on their hosts (Stakman and Harrar 1957). Our ability to perform high-throughput sequencing of these niches has revealed large numbers of microbes forming communities that can affect disease outcomes. More research is required to better understand the composition of organisms in these niches, the interactions among members of these communities, and how the communities impact plant health. A fundamental understanding of the plant microbiome is necessary for successful manipulation for agricultural benefit.

\section{ACKNOWLEDGMENTS}

This work was supported by the Michigan Wheat Program, Michigan State University Plant Science Fellowship, and by the National Science Foundation Long-Term Ecological Research Program (DEB 1637653) at the Kellogg Biological Station and by Michigan State University AgBioResearch. We thank G. Bonito for his suggestions on this manuscript, particularly during the data analysis. 


\section{LITERATURE CITED}

Adhikari, B. N., Bandyopadhyay, R., and Cotty, P. J. 2016. Degeneration of aflatoxin gene clusters in Aspergillus flavus from Africa and North America. AMB Express 6:62.

Altschul, S. F., Gish, W., Miller, W., and Myers, E. W. 1990. Basic local alignment search tool. J. Mol. Biol. 215:403-410.

Arnold, A. E., Maynard, Z., Gilbert, G. S., Coley, P. D., and Kursar, T. A. 2000. Are tropical fungal endophytes hyperdiverse? Ecol. Lett. 3:267-274.

Bakker, M. G., Moorman, T. B., Kaspar, T. C., and Manter, D. K. 2017. Isolation of cultivation-resistant oomycetes, first detected as amplicon sequences, from roots of herbicide-terminated winter rye. Phytobiomes 1:24-35.

Baldwin, T. K., Gaffoor, I., Antoniw, J., Andries, C., Guenther, J., Urban, M., Hallen-Adams, H. E., Pitkin, J., Hammond-Kosack, K. E., and Trail, F. 2010. A partial chromosomal deletion caused by random plasmid integration resulted in a reduced virulence phenotype in Fusarium graminearum. Mol. Plant-Microbe Interact. 23:1083-1096.

Bonito, G., Brenneman, T., and Vilgalys, R. 2011. Ectomycorrhizal fungal diversity in orchards of cultivated pecan (Carya illinoinensis; Juglandaceae). Mycorrhiza 21:601-612.

Bulgarelli, D., Schlaeppi, K., Spaepen, S., van Themaat, E. V. L., and SchulzeLefert, P. 2013. Structure and functions of the bacterial microbiota of plants. Annu. Rev. Plant Biol. 64:807-838.

Chaverri, P., Branco-Rocha, F., Jaklitsch, W., Gazis, R., Degenkolb, T., and Samuels, G. J. 2015. Systematics of the Trichoderma harzianum species complex and the re-identification of commercial biocontrol strains. Mycologia 107:558-590.

Chen, W., Turkington, T. K., Lévesque, C. A., Bamforth, J. M., Patrick, S. K., Lewis, C. T., Chapados, J. T., Gaba, D., Tittlemier, S. A., MacLeod, A., and Gräfenhan, T. 2016. Geography and agronomical practices drive diversification of the epiphytic mycoflora associated with barley and its malt end product in western Canada. Agric. Ecosyst. Environ. 226:43-55.

Cole, J. R., Wang, Q., Fish, J. A., Chai, B., McGarrell, D. M., Sun, Y., Brown, C. T., Porras-Alfaro, A., Kuske, C. R., and Tiedje, J. M. 2013. Ribosomal Database Project: Data and tools for high throughput rRNA analysis. Nucleic Acids Res. 42:D633-D642.

Coleman-Derr, D., Desgarennes, D., Fonseca-Garcia, C., Gross, S., Clingenpeel, S., Woyke, T., North, G., Visel, A., Partida-Martinex, L. P., and Tringe, S. G. 2015. Plant compartment and biogeography affect microbiome composition in cultivated and native Agave species. New Phytol. 209:798-811.

Compant, S., Mitter, B., Colli-Mull, J. G., Gangl, H., and Sessitsch, A. 2011. Endophytes of grapevine flowers, berries, and seeds: Identification of cultivable bacteria, comparison with other plant parts, and visualization of niches of colonization. Microbiol. Ecol. 62:188-197.

Copeland, J. K., Yuan, L., Layeghifard, M., Wang, P. W., and Guttman, D. S. 2015. Seasonal community succession of the phyllosphere microbiome. Mol. Plant-Microbe Interact. 28:274-285.

Crum, J. R., and Collins, H. P. 1995. Soil description: KBS soils. Kellogg Biological Station Long-Term Ecological Research, Michigan State University, Hickory Corners, MI. https://lter.kbs.msu.edu/research/sitedescription-and-maps/soil-description

de Souza, R. S. C., Okura, V. K., Armanhi, J. S. L., Jorrín, B., Lozano, N., da Silva, M. J., González-Guerrero, M., de Araújo, L. M., Verza, N. C., Bagheri, H. C., Imperial, J., and Arruda, P. 2016. Unlocking the bacterial and fungal communities assemblages of sugarcane microbiome. Sci. Rep.-UK 6:1-15.

Donn, S., Kirkegaard, J. A., Perera, G., Richardson, A. E., and Watt, M. 2014. Evolution of bacterial communities in the wheat crop rhizosphere. Environ. Microbiol. 17:610-621.

Edgar, R. C. 2010. Search and clustering orders of magnitude faster than BLAST. Bioinformatics 26:2460-2461.

Edgar, R. C., and Flyvbjerg, H. 2015. Error filtering, pair assembly and error correction for next-generation sequencing reads. Bioinformatics 31:3476-3482.

Edgar, R. C., Haas, B. J., Clemente, J. C., Quince, C., and Knight, R. 2011. UCHIME improves sensitivity and speed of chimera detection. Bioinformatics 27:2194-2200.

Finkel, O. M., Burch, A. Y., Lindow, S. E., Post, A. F., and Belkin, S. 2011. Geographical location determines the population structure in phyllosphere microbial communities of a salt-excreting desert tree. Appl. Environ. Microbiol. 77:7647-7655.

Granzow, S., Kaiser, K., Wemheuer, B., Pfeiffer, B., Daniel, R., Vidal, S., and Wemheuer, F. 2017. The effects of cropping regimes on fungal and bacterial communities of wheat and faba bean in a greenhouse pot experiment differ between plant species and compartment. Front. Microbiol. 8:902.
Grondona, I., Hermosa, R., Tejada, M., Gomis, M. D., Mateos, P. F., Bridge, P. D., Monte, E., and Garcia-Acha, I. 1997. Physiological and biochemical characterization of Trichoderma harzianum, a biological control agent against soilborne fungal plant pathogens. Appl. Environ. Microbiol. 63: 3189-3198.

Hartmann, M., Frey, B., and Mayer, J., Mäder, P., and Widmer, F. 2014. Distinct soil microbial diversity under long-term organic and conventional farming. ISME J. 9:1177-1194.

Huang, Y., Kuang, Z., Wang, W., and Cao, L. 2016. Exploring potential bacterial and fungal biocontrol agents transmitted from seeds to sprouts of wheat. Biol. Control 98:27-33.

Hubbard, M., Germida, J., and Vujanovic, V. 2012. Fungal endophytes improve wheat seed germination under heat and drought stress. Botany 90 : 137-149.

Karlsson, I., Friberg, H., Kolseth, A. K., and Steinberg, C. 2017. Organic farming increases richness of fungal taxa in the wheat phyllosphere. Mol. Ecol. 2017:1-13

Kelly, B. J., Gross, R., Bittinger, K., Sherrill-Mix, S., Lewis, J. D., Collman, R. G., Bushman, F. D., and Li, H. 2015. Power and sample-size estimation for microbiome studies using pairwise distances and PERMANOVA. Bioinformatics 31:2461-2468.

Knief, C., Delmotte, N. E. L., Chaffron, S., Stark, M., Innerebner, G., Wassmann, R., von Mering, C., and Vorholt, J. A. 2011. Metaproteogenomic analysis of microbial communities in the phyllosphere and rhizosphere of rice. ISME J. 6:1378-1390.

Kõljalg, U., Nilsson, R. H., Abarenkov, K., Tedersoo, L., Taylor, A. F. S., and Bahram, M., Bates, S. T., Bruns, T. D., Bengtsson-Palme, J., Callaghan, T. M., Douglas, N., Drenkhan, T., Eberhardt, U., Duenas, M., Grebenc, T., Griffith, G. W., Hartmann, M., Kirk, P. M., Kohout, P., Larsson, E., Lindahl, B. D., Lücking, R., Martín, M. P., Matheny, P. B., Nguyen, N. H., Niskanen, T., Oja, J., Peay, K. G., Peintner, U., Peterson, M., Põldmaa, K., Saag, L., Saar, I., Schüßler, A., Scott, J. A., Senés, C., Smith, M. E., Suija, A., Taylor, D. L., Telleria, M. T., Weiss, M., and Larsson, K.-H. 2013. Towards a unified paradigm for sequence-based identification of fungi. Mol. Ecol. 22: 5271-5277.

Kozich, J. J., Westcott, S. L., Baxter, N. T., Highlander, S. K., and Schloss, P. D. 2013. Development of a dual-index sequencing strategy and curation pipeline for analyzing amplicon sequence data on the MiSeq Illumina sequencing platform. Appl. Environ. Microbiol. 79:5112-5120.

Kwaśna, H., Bateman, G. L., and Ward, E. 2010. Microbiota in wheat roots evaluated by cloning of ITS $1 / 2$ rDNA and sequencing. J. Phytopathol. 158:278-287.

Lauber, C. L., Ramirez, K. S., Aanderud, Z., Lennon, J., and Fierer, N. 2013. Temporal variability in soil microbial communities across land-use types. ISME J. 7:1641-1650.

Lenc, L., Kwaśna, H., Sadowski, C., and Grabowski, A. 2014. Microbiota in wheat roots, rhizosphere and soil in crops grown in organic and other production systems. J. Phytopathol. 163:245-263.

Li, R., Khafipour, E., Krause, D. O., Entz, M. H., de Kievit, T. R., and Fernando, W. G. D. 2012. Pyrosequencing reveals the influence of organic and conventional farming systems on bacterial communities. PLoS One 7:e51897.

Love, M. I., Huber, W., and Anders, S. 2014. Moderated estimation of fold change and dispersion for RNA-seq data with DESeq2. Genome Biol. 15: 550 .

Mahoney, A. K., Yin, C., and Hulbert, S. H. 2017. Community structure, species variation, and potential functions of rhizosphere-associated bacteria of different winter wheat (Triticum aestivum) cultivars. Front. Plant Sci. 8:132.

McMullen, M., Bergstrom, G., De Wolf, E., Dill-Macky, R., Hershman, D., Shaner, G., and Van Sanford, D. 2012. A unified effort to fight an enemy of wheat and barley: Fusarium head blight. Plant Dis. 96:1712-1728.

McMurdie, P. J., and Holmes, S. 2013. phyloseq: An R package for reproducible interactive analysis and graphics of microbiome census data. PLoS One 8:e61217.

McMurdie, P. J., and Holmes, S. 2014. Waste not, want not: why rarefying microbiome data is inadmissible. PLOS Comput. Biol. 10:e1003531.

Mendes, L. W., Kuramae, E. E., Navarrete, A. A. C. A., van Veen, J. A., and Tsai, S. M. 2014. Taxonomical and functional microbial community selection in soybean rhizosphere. ISME J. 8:1577-1587.

Mousa, W. K., Shearer, C., Limay-Rios, V., and Ettinger, C. L. 2016. Root-hair endophyte stacking in finger millet creates a physicochemical barrier to trap the fungal pathogen Fusarium graminearum. Nature Microbiol. 1:16167.

Nettles, R., Watkins, J., Ricks, K., Boyer, M., Licht, M., Atwood, L. W., Peoples, M., Smith, R. G., Mortensen, D. A., and Koide, R. T. 2016. Influence of pesticide seed treatments on rhizosphere fungal and bacterial communities 
and leaf fungal endophyte communities in maize and soybean. Appl. Soil Ecol. 102:61-69.

Ofek, M., Voronov-Goldman, M., Hadar, Y., and Minz, D. 2013. Host signature effect on plant root-associated microbiomes revealed through analyses of resident vs. active communities. Environ. Microbiol. 16:2157-2167.

Oksanen, J., Blanchet, F. G., Friendly, M., Kindt, R., Legendre, P., and McGlinn, D. 2016. vegan: Community Ecology Package. https://CRAN.Rproject.org/web/packages/vegan/index.html

Peay, K. G., Kennedy, P. G., and Talbot, J. M. 2016. Dimensions of biodiversity in the Earth mycobiome. Nat. Rev. Microbiol. 14:434-447.

Peiffer, J. A., Spor, A., Koren, O., and Jin, Z. 2013. Diversity and heritability of the maize rhizosphere microbiome under field conditions. Proc. Natl. Acad. Sci. USA 110:6548-6553.

R Core Team. 2016. R: A language and environment for statistical computing. R Core Team, Vienna, Austria. https://www.R-project.org/

Rascovan, N., Carbonetto, B., Perrig, D., Díaz, M., Canciani, W., Abalo, M., Alloati, J., González-Ant, G., and Vazquez, M. P. 2016. Integrated analysis of root microbiomes of soybean and wheat from agricultural fields. Sci. Rep.-UK 6:1-12.

Rastogi, G., Coaker, G. L., and Leveau, J. H. J. 2013. New insights into the structure and function of phyllosphere microbiota through high-throughput molecular approaches. FEMS Microbiol. Lett. 348:1-10.

Reasoner, D. J., and Geldreich, E. E. 1985. A new medium for the enumeration and subculture of bacteria from potable water. Appl. Environ. Microbiol. 49:1-7.

Rideout, J. R., He, Y., Navas-Molina, J. A., Walters, W. A., Ursell, L. K., Gibbons, S. M., Chase, J., McDonald, D., Gonzalez, A., Robbins-Pianka, A., Clemente, J. C., Gilbert, J., Huse, S. M., Zhou, H. W., Knight, R., and Caporaso, J. G. 2014. Subsampled open-reference clustering creates consistent, comprehensive OTU definitions and scales to billions of sequences. PeerJ 2:e545.

Robertson, P. G. 2015. The Ecology of Agricultural Landscapes: Long-Term Research on the Path to Sustainability. P. G. Robertson, S. K. Hamilton, and J. E. Doll, eds. Oxford University Press, Oxford, UK.

Rodriguez, R. J., Henson, J., Van Volkenburgh, E., Hoy, M., Wright, L., Beckwith, F., Kim, Y. O., and Redman, R. S. 2008. Stress tolerance in plants via habitat-adapted symbiosis. ISME J. 2:404-416.

Rodriguez, R. J., White, J. F., Jr., Arnold, A. E., and Redman, R. S. 2009. Fungal endophytes: Diversity and functional roles. New Phytol. 182:314-330.

Ruinen, J. 1956. Occurrence of Beijerinckia species in the 'phyllosphere'. Nature 177:220-221.

Shade, A., McManus, P. S., and Handelsman, J. 2013. Unexpected diversity during community succession in the apple flower microbiome. MBio 4:e00602-e00612.
Sharma, K., Mishra, A. K., and Misra, R. S. 2009. Morphological, biochemical and molecular characterization of Trichoderma harzianum isolates for their efficacy as biocontrol agents. J. Phytopathol. 157:51-56.

Sieber, T., Riesen, T. K., Müller, E., and Fried, P. M. 1988. Endophytic fungi in four winter wheat cultivars (Triticum aestivum L.) differing in resistance against Stagonospora nodorum (Berk.) Cast. \& Germ.= Septoria nodorum (Berk.) Berk. J. Phytopathol. 122:289-306.

Slininger, P. J., Schisler, D. A., Shea-Andersh, M. A., Sloan, J. M., Woodell, L. K., Frazier, M. J., and Olsen, N. L. 2010. Multi-strain co-cultures surpass blends for broad spectrum biological control of maladies of potatoes in storage. Biocontrol Sci. Technol. 20:763-786.

Stakman, E. C., and Harrar, J. G. 1957. Principles of Plant Pathology. The Ronald Press Company, New York.

Suay, I., Arenal, F., Asensio, F. J., Basilio, A., Cabello, M. A., Díez, M. T., García, J. B., del Val, A., Gorrochategui, J., Hernandez, P., Pelaez, F., and Vicente, M. F. 2000. Screening of basidiomycetes for antimicrobial activities. Antonie van Leeuwenhoek 78:129-140.

Trail, F., and Common, R. 2000. Perithecial development by Gibberella zeae: A light microscopy study. Mycologia 92:130-138.

USDA. 2016. Crop Production 2015 Summary (January 2016). USDA, National Agricultural Statistics Service.

Vorholt, J. A. 2012. Microbial life in the phyllosphere. Nat. Rev. Microbiol. 10:828-840.

Wang, Q., Garrity, G. M., Tiedje, J. M., and Cole, J. R. 2007. Naive Bayesian classifier for rapid assignment of rRNA sequences into the new bacterial taxonomy. Appl. Environ. Microbiol. 73:5261-5267.

Wegulo, S. N., Baenziger, P. S., Nopsa, J. H., Bockus, W. W., and HallenAdams, H. 2015. Management of Fusarium head blight of wheat and barley. Crop Prot. 73:100-107.

Wickham, H. 2009. ggplot2: Elegant Graphics for Data Analysis. Springer, New York.

Xue, K., Wu, L., Deng, Y., He, Z., Van Nostrand, J., Robertson, P. G., Schmidt, T. M., and Zhou, J. 2013. Functional gene differences in soil microbial communities from conventional, low-input, and organic Farmlands. Appl. Environ. Microbiol. 79:1284-1292.

Yin, C., Mueth, N., Hulbert, S., Schlatter, D., Paulitz, T. C., Schroeder, K., Prescott, A., and Dhingra, A. 2017. Bacterial communities on wheat grown under long-term conventional tillage and no-till in the Pacific Northwest of the United States. Phytobiomes 1:83-90.

Zadoks, J. C., Chang, T. T., and Konzak, C. F. 1974. A decimal code for the growth stages of cereals. Weed Res. 14:415-421. 